\section{Encephalitis Associated With Hemophagocytic Lymphohistiocytosis Secondary to Immune Checkpoint Inhibitors: An Unfamiliar Spin-Off}

Ghulam Ghous ${ }^{1}$, Hafiz Muhammad Hassan Shoukat ${ }^{2}$, Zahid Ijaz Tarar ${ }^{3}$, Muhammad Usman Zafar ${ }^{4}$, Joseph W. McGreevy ${ }^{3}$

1. Internal Medicine, University of Missouri Columbia, Columbia, USA 2. Internal Medicine, Premier Health/Wright State University, Dayton, USA 3. Internal Medicine, University of Missouri, Columbia, USA 4. Hospital Medicine, Lehigh Valley Health Network, Allentown, USA

Corresponding author: Ghulam Ghous,ghulam.ghous77@yahoo.com
Review began 06/03/2021 Review ended 06/20/2021 Published 06/30/2021

() Copyright 2021

Ghous et al. This is an open access article distributed under the terms of the Creative Commons Attribution License CC-BY 4.0., which permits unrestricted use, distribution, and reproduction in any medium, provided the original author and source are credited.

\begin{abstract}
Checkpoint inhibitors (CPI) have become mainstream in standard therapy in various tumors, especially in malignant melanoma. Despite their widespread beneficial effects, these inhibitors are also notorious for immune-related adverse events (irAEs). Hemophagocytic lymphohistiocytosis (HLH) is an aggressive and life-threatening syndrome of excessive immune activation. We report a case of a 33-year-old male having a history of metastatic melanoma on immunotherapy (status post two cycles of ipilimumab/nivolumab) admitted for persistent fever and elevated liver enzymes. Additional work showed anemia, thrombocytopenia, hypertriglyceridemia, and hyperferritinemia which meet the diagnostic criteria of histiocyte society HLH-2004. The patient was effectively treated with oral prednisone. Moreover, further complications encompassed slurred speech, word-finding difficulties, ataxia, and lower extremity hyperreflexia concerning for autoimmune encephalitis. He was treated with high-dose IV methylprednisolone (1 gram/day for 3 days) with improvement in symptoms. Autoimmune encephalitis associated with HLH can be fatal - high-dose IV methylprednisolone should be considered, but this avenue still needs to be explored.
\end{abstract}

Categories: Neurology, Oncology, Hematology

Keywords: encephalitis, hlh, steroids, melanoma, ici

\section{Introduction}

Immune checkpoint inhibitors (ICI), such as anti-programmed death-1 (PD-1) and anti-cytotoxic Tlymphocyte-associated antigen 4 (CTLA-4) antibodies, have shown widespread effectiveness in treating many cancers particularly malignant melanoma. On the downside, ICI therapy is associated with immunerelated adverse events (irAEs) including pneumonitis, hepatitis, colitis, and endocrinopathies such as adrenal insufficiency, thyroid dysfunction, hypopituitarism, and type 1 diabetes mellitus [1]. However new and rare irAEs, including hematologic toxicities, are still an understudied territory, particularly in the case of dual ICI therapy. Management of hematologic irAEs such as thrombotic thrombocytopenic purpura/hemolytic uremic syndrome, aplastic anemia, autoimmune hemolytic anemia, and immune thrombocytopenia have been elaborated in recent literature [2]. Many case reports have been published lately describing hemophagocytic lymphohistiocytosis (HLH) in solid tumors secondary to ICI [3-9]. This case report is about a new finding of HLH associated encephalitis in a malignant melanoma patient treated with nivolumab/ipilimumab.

\section{Case Presentation}

A 33-year-old male having a history of malignant melanoma with metastatic disease to the bones on immunotherapy (status post 2 cycles of ipilimumab/nivolumab) admitted from the emergency department for persistent fever and elevated liver enzymes. The patient reported daily fever as high as $103.5^{\circ} \mathrm{F}$ (taken orally) for four days associated with two episodes of non-bloody emesis the day before admission. He had overall poor appetite but no abdominal pain or diarrhea. Headaches occurred only with fever. He denied any body aches, neck stiffness, photophobia. The patient was seen in urgent care three days ago with both negative rapid influenza and COVID-19 test along with no acute findings on chest X-ray. He was also seen in another hospital three days ago for the same symptoms, had blood cultures drawn, and discharged home on oral antibiotics. On physical examination, the patient had a temperature of $103^{\circ} \mathrm{F}$, pulse $88 \mathrm{bpm}, \mathrm{BP}$ 134/81 $\mathrm{mmHg}$, and oxygen saturation 96\%. Respiratory, gastrointestinal, and neurological examinations were normal.

The initial investigations showed platelet count 94,000 per microliter (normal 150,000 to 400,000 per microliter), total bilirubin $3.56 \mathrm{mg} / \mathrm{dl}$ (normal $0.00-1.60 \mathrm{mg} / \mathrm{dl}$ ), alkaline phosphatase 409 units/L (normal 40-129 units/L), aspartate aminotransferase (AST) 316 units/L (normal less than 40 units/L), alanine transaminase (ALT) 363 units/L (normal 10-50). The right upper quadrant ultrasound demonstrated mild 


\section{Cureus}

periportal edema, no evidence of gallstones or cholecystitis, non-dilated bile ducts. The patient was empirically started on piperacillin/tazobactam and vancomycin after sending two sets of blood cultures. Viral respiratory pathogen panel by polymerase chain reaction (PCR), autoimmune hepatitis work up, and viral hepatitis panel were also sent and all came back negative. The final blood culture report obtained from outside the hospital did not show any growth after four days. The patient continued to spike fever $101-103^{\circ} \mathrm{F}$ despite being on antibiotics for 24 hours. CT chest/abdomen/pelvis and MRI liver also obtained in the setting of persistent fever to look for occult infection and metastatic lesions in the liver but did not show any acute pathology other than hepatomegaly measuring $23 \mathrm{~cm}$ (normal less than $16 \mathrm{~cm}$ ) and splenomegaly measuring $24 \mathrm{~cm}$ (normal less than $12 \mathrm{~cm}$ ) (Figure 1). HIV, cytomegalovirus PCR, and Epstein-Barr virus PCR came negative. A hepatologist was consulted for elevated liver enzymes and recommended liver biopsy and prednisone $1 \mathrm{mg} / \mathrm{kg}$ daily for possible immunotherapy-induced hepatitis. Liver biopsy showed multifocal lobular lymphocytic infiltrates within hepatic sinusoids (Figure $2 \mathrm{~A}$ ), and small mature lymphocytes long with rare eosinophils causing moderate portal inflammation, confirming immunotherapy-induced hepatitis (Figure 2B).

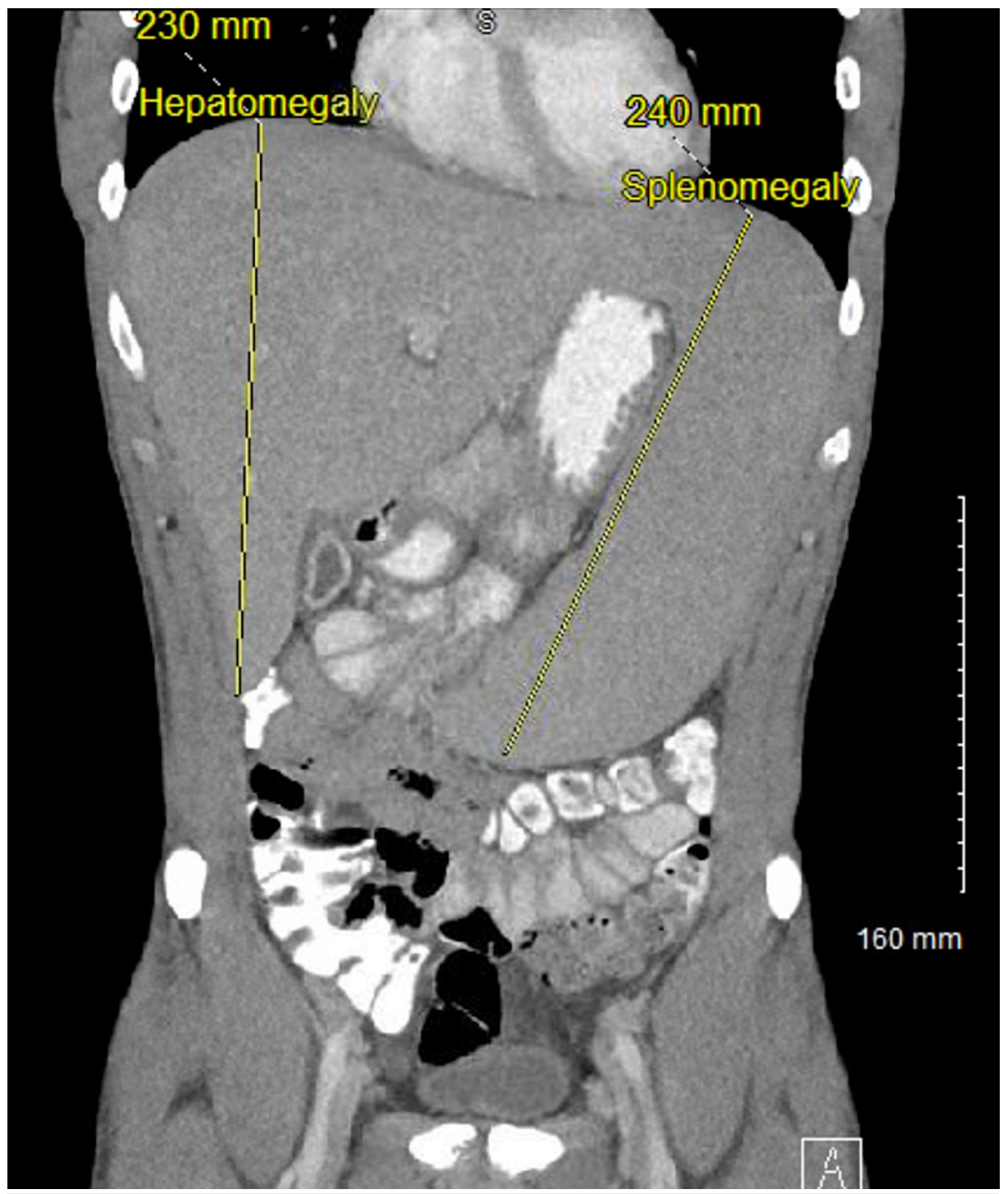

FIGURE 1: Contrast-enhanced CT abdomen and pelvis (coronal view) demonstrating hepatosplenomegaly. 


\section{Cureus}
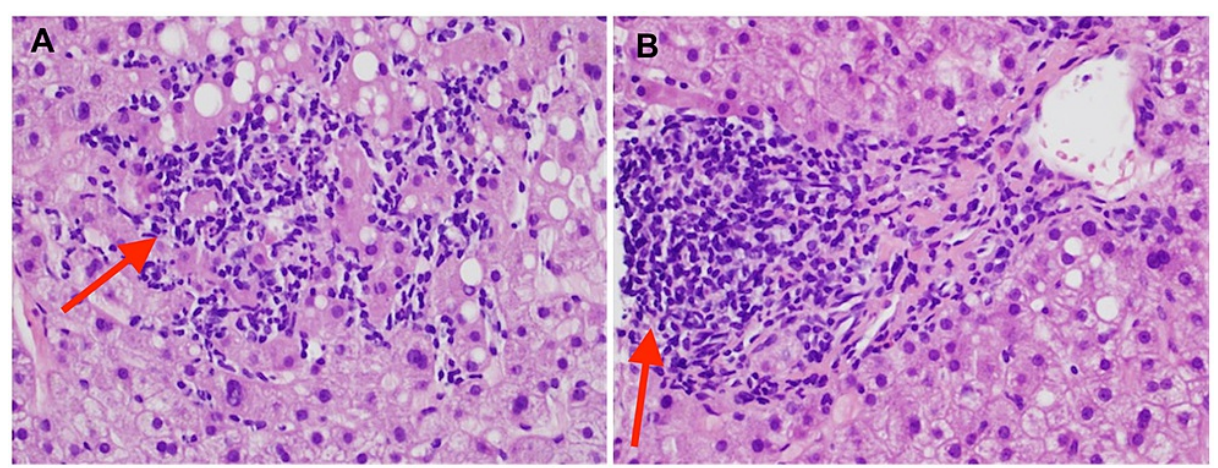

FIGURE 2: Photomicrographs of liver biopsy (H\&E, 40x).

(A) showing patchy lobular lymphocytic inflammation with mild macrovascular steatosis background. The presence of multifocal lobular lymphocytic inflammation (acute lobular hepatitis pattern of injury) is consistent with immunotherapy-associated liver toxicity.

(B) Portal area showing focal moderate portal inflammation mixed with small mature lymphocytes and rare eosinophils suggestive of immune checkpoint inhibitors hepatotoxicity.

On day 4 of admission, the patient started to have slurred speech, word-finding difficulty, ataxia, and lower extremity hyperreflexia concerning for autoimmune encephalitis. Neurology was consulted and recommended paraneoplastic antibodies, MRI brain, and lumbar puncture. Serum ferritin, triglycerides, fibrinogen, and soluble IL-2 receptor (CD25) were also sent for possible HLH with associated encephalitis. Ferritin level came back $3446 \mathrm{ng} / \mathrm{ml}$, triglycerides $525 \mathrm{mg} / \mathrm{dl}$, fibrinogen $145 \mathrm{mg} / \mathrm{dl}$, and soluble IL-2 receptor (CD25) 1405 pg/ml (age-related normal 1033 pg/ml). MRI brain with contrast showed no acute intracranial abnormalities. Serum ammonia level was also within normal limits. Cerebrospinal fluid (CSF) analysis showed elevated protein $199 \mathrm{mg} / \mathrm{dl}$ (normal 15-45 mg/dl) with normal WBC count. Oligoclonal bands in serum and CSF were 6 and the IgG index was 0.67 (normal 0.32-0.60). Paraneoplastic antibody panel (type 1 antineuronal nuclear antibody [ANNA-1], type 2 antineuronal nuclear antibody [ANNA-2], Purkinje cell cytoplasmic antibody type 1 [PCA-1], collapsin response-mediator protein-5 [CRMP-5] antibody, amphiphysin antibody, anti-N-methyl-D-aspartate [NMDA] receptor antibody, gamma-aminobutyric acid type A [GABA-A] and type B [GABA-B] receptor antibodies, contactin-associated protein-like 2 [Caspr2] antibody) was normal except for mildly elevated glutamic acid decarboxylase (GAD) antibody. GAD autoantibodies (GADAs) are associated with various neurologic conditions, such as stiff person syndrome, cerebellar ataxia, limbic encephalitis, myasthenia gravis, and epilepsy. Elevated GAD antibodies in our case were suggestive of encephalitis.

The patient was started on prednisone $1 \mathrm{mg} / \mathrm{kg}$ daily for immunotherapy-induced hepatitis. Prednisone was increased to $2 \mathrm{mg} / \mathrm{kg}$ on day 2 for worsening hyperbilirubinemia. The patient's fever resolved 24 hours after starting steroids. Antibiotics were stopped (absence of infection). Liver enzymes also started to improve. Few days into admission, the patient started to develop neurological symptoms and after the aforementioned workup, he was diagnosed with encephalitis associated with HLH secondary to ipilimumab/nivolumab. Methylprednisolone was increased to 1 gram daily for three days with improvement in all neurological symptoms and the patient was discharged home on prednisone taper.

The patient's fever settled a day after starting steroids and all neurological symptoms improved with highdose IV methylprednisolone (1 gram daily for three days). Liver enzymes also started to improve, and he was discharged on prednisone $2 \mathrm{mg} / \mathrm{kg}$ daily. AST and bilirubin normalized after 10 days (Figure 3 and Figure 4). ALT also started to improve but plateaued (Figure 4). The patient was advised to continue prednisone 2 $\mathrm{mg} / \mathrm{kg}$ daily till ALT level gets orderly. At eight weeks follow-up, ALT normalized, and prednisone was gradually tapered over the next four weeks. The patient had no new neurological symptoms throughout treatment. 


\section{Cureus}

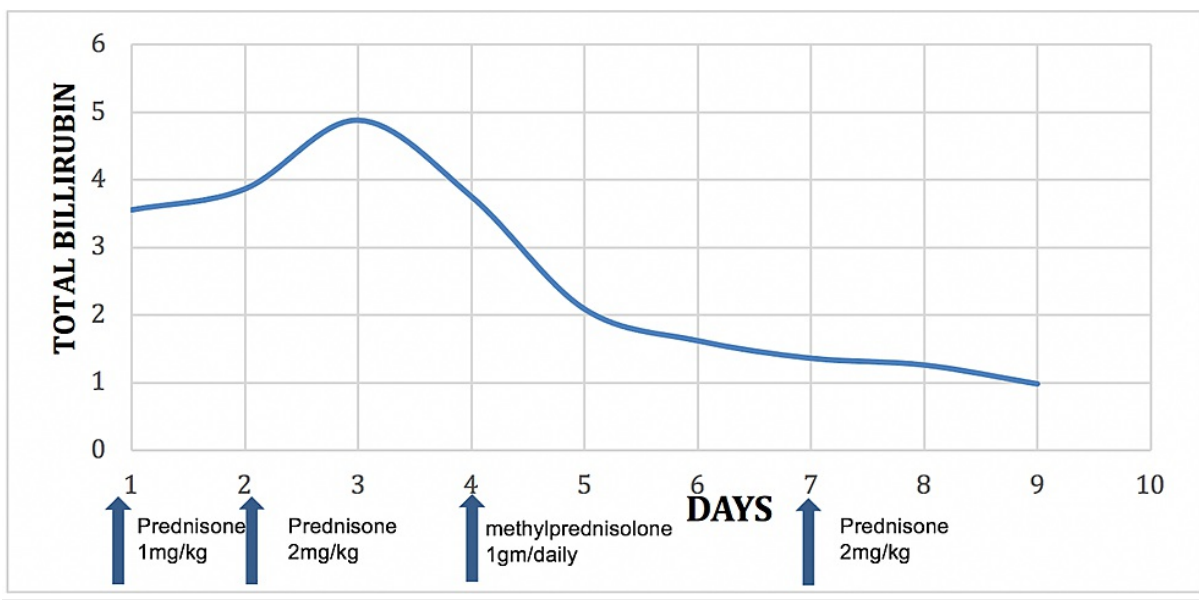

FIGURE 3: Total bilirubin levels in response to steroids.

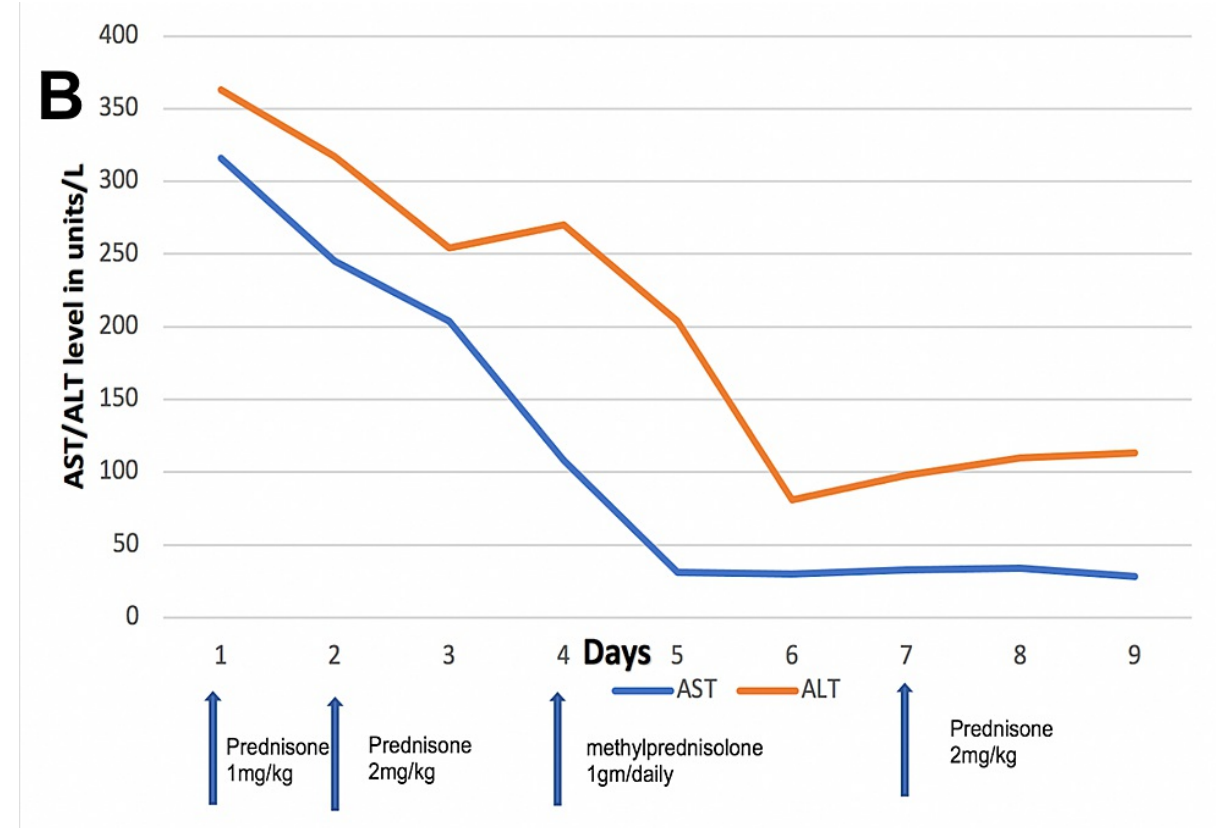

FIGURE 4: AST and ALT levels in response steroids.

ALT, alanine transaminase; AST, aspartate aminotransferase.

\section{Discussion}

Hemophagocytic lymphohistiocytosis (HLH) is a progressively fatal syndrome of hyperactive immune system and requires prompt attention. Diagnostic criteria for HLH is listed below (Table 1) [3-5]. 


\section{Cureus}

The diagnosis of HLH can be established if either 1 or 2 are fulfilled:

1. A molecular diagnosis consistent with HLH: pathological mutations of PRF1, UNC13D, STXBP1, RAB27A, STX11, SH2D1A, or XIAP

2. Diagnostic criteria for HLH fulfilled (5 out of the 8 criteria below): ${ }^{a}$

Fever $38.5^{\circ} \mathrm{C}\left(101.3^{\circ} \mathrm{F}\right)$ or more

Splenomegaly

Cytopenias (affecting at least 2 of 3 cell lineages in the peripheral blood):

Hemoglobin

Platelets $<100 \times 18 / L$

Neutrophils $<1.0 \times 18 / \mathrm{L}$

Platelets $<100 \times 18 / L$

Hypertriglyceridemia and/or hypofibrinogenemia

Fasting triglycerides $\geq 3.0 \mathrm{mmol} / \mathrm{L}$ (i.e., $\geq 265 \mathrm{mg} / \mathrm{dL}$ )

Fibrinogen $\leq 1.5 \mathrm{~g} / \mathrm{L}$

Hemophagocytosis in bone marrow or spleen or lymph nodes or liver

Low or absent NK-cell activity

Ferritin $\geq 500 \mathrm{mg} / \mathrm{L}$

Soluble CD25 (i.e., soluble IL-2 receptor) $\geq 2400 \mathrm{U} / \mathrm{mL}^{\mathrm{b}}$

\section{TABLE 1: HLH-2004 diagnostic criteria.}

aSupportive criteria include neurologic symptoms, cerebrospinal pleocytosis, conjugated hyperbilirubinemia and transaminitis, hypoalbuminemia, hyponatremia, elevated D-dimers, and lactate dehydrogenase. The absence of hemophagocytosis (in the bone marrow) does not exclude a diagnosis of $\mathrm{HLH}$

bNew data show normal variation by age. Level should be compared with age-related norms.

HLH, Hemophagocytic lymphohistiocytosis; NK-cell, Natural killer cells.

North American Consortium for Histiocytosis (NACHO) advocate against the terms "primary HLH" and "secondary HLH," owing to the fact that these terms cause confusion as both primary and secondary HLH can be activated by infections or other immune-related pathologies, and gene mutations can occur in any person regardless of age and family history [6]. NACHO prefers the following terminologies [6]:

HLH syndrome - A condition of pathologic immune activation that is often associated with genetic defects of lymphocyte cytotoxicity. This includes all conditions meeting consensus diagnostic criteria HLH disease HLH syndrome in which the distinctive immune activation is the core problem and that would benefit from HLH-directed immunosuppressive therapies such as HLH observed after immune-activating therapies (iatrogenic HLH, also called cytokine release syndrome), familial HLH with clear genetic etiology, HLH associated with malignancy, HLH associated with rheumatologic conditions (also called MAS).

HLH disease mimics - Other disorders that resemble HLH syndrome but are caused by other conditions and that would not benefit from immunosuppressive therapy or require entirely different treatments.

Pathophysiology of HLH comprises abnormal working of macrophages, natural killer cells (NK cells), cytotoxic lymphocytes (CTLs), and altered numbers of CD4 and CD8 lymphocyte subsets [7]. HLH induces organ failure because of tissue damage as an outcome of over activation of macrophages resulting in over secretion of cytokines. CTLs or NK cells are not able to remove activated macrophages leading to enhanced activity of macrophages and raised values of various cytokines such as interferon-gamma. HLH patients have diminished cytotoxic activity of CTLs and NK cells, associated with enhanced macrophage activation. This stimulated production of cytokines is considered a major perpetrator of tissue injury and multi-organ 


\section{Cureus}

failure leading to high mortality [8-10].

The onset of an acute episode of HLH can trigger because of infection or modification in immune balance which can occur either due to immune activation or immune deficiency. Immune activation from an infection is a common trigger [11]. The immune checkpoint inhibitors can also cause immune activation and $\mathrm{HLH}$, but the incidence has not been defined.

We found a total of 10 cases of ICI-associated HLH in malignant melanoma including nivolumab [12], pembrolizumab [5,13], combined pembrolizumab, and ipilimumab [14], combined ipilimumab and nivolumab [12,14-16]. Six of ten patients had no other immune-related toxicities while 4 others showed thyroiditis [16], hepatic cytolysis, lymphocytic meningitis, and colitis [14]. All 10 patients received steroids in the 0.5 to $2 \mathrm{mg} / \mathrm{kg}$ range. Out of those, three were given extra immunosuppression, one with mycophenolate mofetil [16], and one with etoposide and tocilizumab [14], and the other with etoposide [14] (Table 2). 


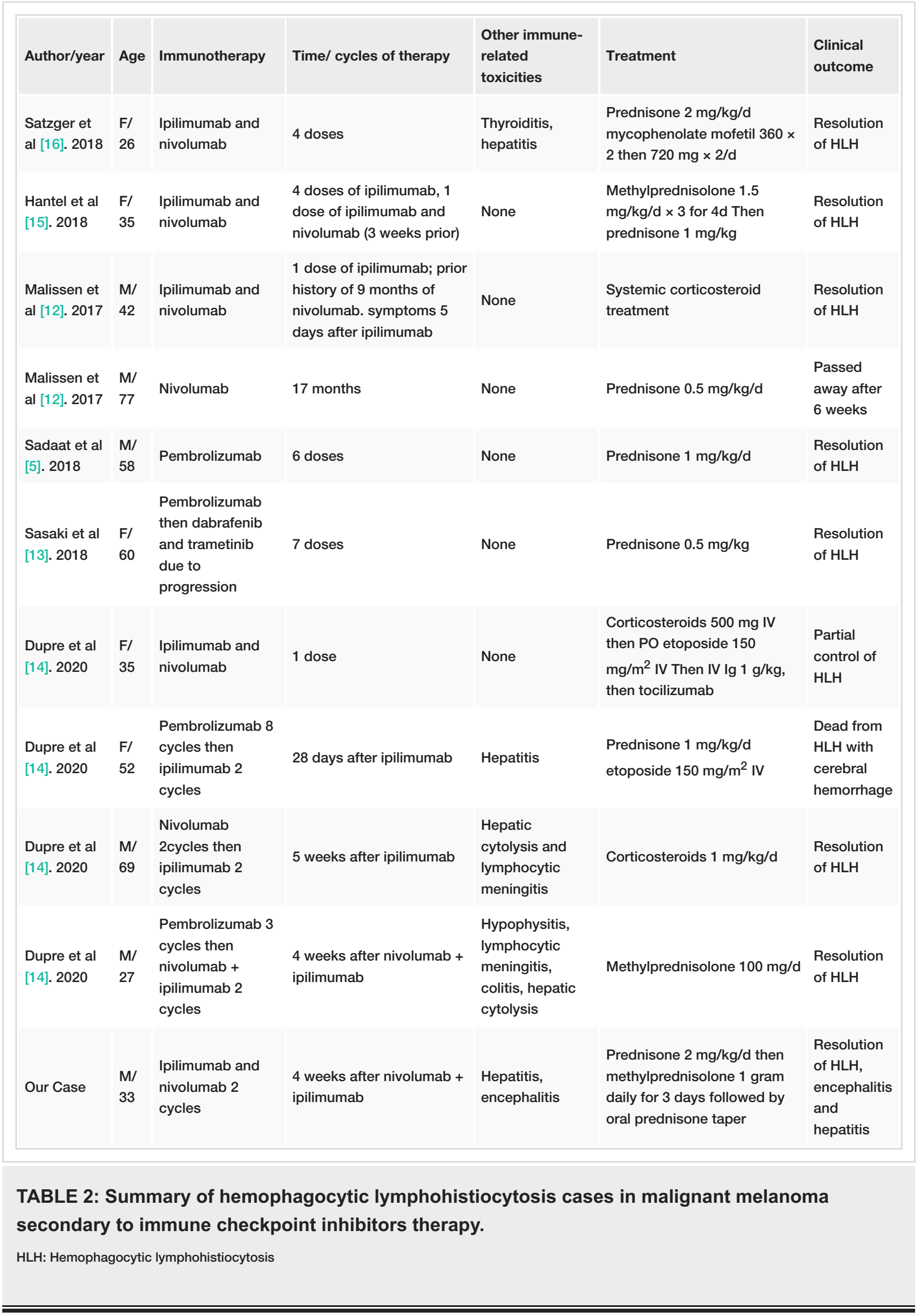

We report the first case of encephalitis associated with HLH in the setting of ICI for malignant melanoma. Initially, we treated the patient with prednisone $1 \mathrm{mg} / \mathrm{kg}$ for HLH but when he showed symptoms of encephalitis including slurred speech, word-finding difficulties, ataxia, and lower extremity hyperreflexia we increased steroids to methylprednisolone to 1 gram daily and treated the patient for three days with 
complete resolution of encephalitis symptoms.

\section{Conclusions}

This is the first reported case of encephalitis associated with HLH secondary to combination ICI therapy in the setting of malignant melanoma treated with high dose methylprednisolone (1gm/daily for 3 days). HLHassociated encephalitis should be suspected in a patient exhibiting neurological symptoms other than HLH features, high-dose IV methylprednisolone $1 \mathrm{gm} /$ daily should be considered but further evidence is required.

\section{Additional Information \\ Disclosures}

Human subjects: Consent was obtained or waived by all participants in this study. Conflicts of interest: In compliance with the ICMJE uniform disclosure form, all authors declare the following: Payment/services info: All authors have declared that no financial support was received from any organization for the submitted work. Financial relationships: All authors have declared that they have no financial relationships at present or within the previous three years with any organizations that might have an interest in the submitted work. Other relationships: All authors have declared that there are no other relationships or activities that could appear to have influenced the submitted work.

\section{References}

1. Topalian SL, Hodi FS, Brahmer JR, et al.: Safety, activity, and immune correlates of anti-PD-1 antibody in cancer. N Engl J Med. 2012, 366:2443-54. 10.1056/NEJMoa1200690

2. Brahmer JR, Lacchetti C, Thompson JA: Management of immune-related adverse events in patients treated with immune checkpoint inhibitor therapy: American Society of Clinical Oncology Clinical Practice Guideline summary. J Oncol Pract. 2018, 14:247-9. 10.1200/JOP.18.00005

3. Jordan MB, Allen CE, Weitzman S, Filipovich AH, McClain KL: How I treat hemophagocytic lymphohistiocytosis. Blood. 2011, 118:4041-52. 10.1182/blood-2011-03-278127

4. Bergsten E, Horne A, Aricó M, et al.: Confirmed efficacy of etoposide and dexamethasone in HLH treatment: long-term results of the cooperative HLH-2004 study. Blood. 2017, 130:2728-38. 10.1182/blood-2017-06788349

5. Sadaat M, Jang S: Hemophagocytic lymphohistiocytosis with immunotherapy: brief review and case report. J Immunother Cancer. 2018, 6:49. 10.1186/s40425-018-0365-3

6. Jordan MB, Allen CE, Greenberg J, et al.: Challenges in the diagnosis of hemophagocytic lymphohistiocytosis: Recommendations from the North American Consortium for Histiocytosis (NACHO). Pediatr Blood Cancer. 2019, 66:e27929. 10.1002/pbc.27929

7. Dalal BI, Vakil AP, Khare NS, Wang SY, Richards MJ, Chen LY: Abnormalities of the lymphocyte subsets and their immunophenotype, and their prognostic significance in adult patients with hemophagocytic lymphohistiocytosis. Ann Hematol. 2015, 94:1111-7. 10.1007/s00277-015-2350-y

8. Pachlopnik Schmid J, Côte M, Ménager MM, et al.: Inherited defects in lymphocyte cytotoxic activity . Immunol Rev. 2010, 235:10-23. 10.1111/j.0105-2896.2010.00890.x

9. Risma K, Jordan MB: Hemophagocytic lymphohistiocytosis: updates and evolving concepts. Curr Opin Pediatr. 2012, 24:9-15. 10.1097/MOP.0b013e32834ec9c1

10. Stepp SE, Dufourcq-Lagelouse R, Le Deist F, et al.: Perforin gene defects in familial hemophagocytic lymphohistiocytosis. Science. 1999, 286:1957-9. 10.1126/science.286.5446.1957

11. Serrão T, Dias A, Nunes P, Figueiredo A: An uncommon presentation of EBV-driven HLH. Primary or secondary? An ongoing dilemma. BMJ Case Rep. 2015, 2015: 10.1136/bcr-2015-209615

12. Malissen N, Lacotte J, Du-Thanh A, Gaudy-Marqueste C, Guillot B, Grob JJ: Macrophage activation syndrome: a new complication of checkpoint inhibitors. Eur J Cancer. 2017, 77:88-9. 10.1016/j.ejca.2017.02.016

13. Sasaki K, Uehara J, Iinuma S, Doi H, Honma M, Toki Y, Ishida-Yamamoto A: Hemophagocytic lymphohistiocytosis associated with dabrafenib and trametinib combination therapy following pembrolizumab administration for advanced melanoma. Ann Oncol. 2018, 29:1602-3. 10.1093/annonc/mdy175

14. Dupré A, Michot JM, Schoeffler A, et al.: Haemophagocytic lymphohistiocytosis associated with immune checkpoint inhibitors: a descriptive case study and literature review. Br J Haematol. 2020, 189:985-92. 10.1111/bjh.16630

15. Hantel A, Gabster B, Cheng JX, Golomb H, Gajewski TF: Severe hemophagocytic lymphohistiocytosis in a melanoma patient treated with ipilimumab + nivolumab. J Immunother Cancer. 2018, 6:73. 10.1186/s40425018-0384-0

16. Satzger I, Ivanyi P, Länger F, et al.: Treatment-related hemophagocytic lymphohistiocytosis secondary to checkpoint inhibition with nivolumab plus ipilimumab. Eur J Cancer. 2018, 93:150-3.

10.1016/j.ejca.2018.01.063 